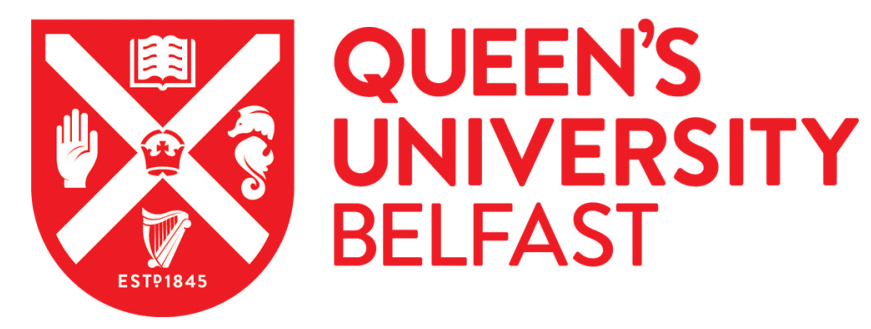

\title{
Propionibacterium acnes and lipopolysaccharide induce the expression of antimicrobial peptides and proinflammatory cytokines/chemokines in human sebocytes
}

Nagy, I., Pivarcsi, A., Kis, K., Koreck, A., Bodai, L., McDowell, A., Seltmann, H., Patrick, S., Zouboulis, C. C., \& Kemeny, L. (2006). Propionibacterium acnes and lipopolysaccharide induce the expression of antimicrobial peptides and proinflammatory cytokines/chemokines in human sebocytes. Microbes and Infection, 8(8), 21952205.

Published in:

Microbes and Infection

Queen's University Belfast - Research Portal:

Link to publication record in Queen's University Belfast Research Portal

\section{General rights}

Copyright for the publications made accessible via the Queen's University Belfast Research Portal is retained by the author(s) and / or other copyright owners and it is a condition of accessing these publications that users recognise and abide by the legal requirements associated with these rights.

Take down policy

The Research Portal is Queen's institutional repository that provides access to Queen's research output. Every effort has been made to ensure that content in the Research Portal does not infringe any person's rights, or applicable UK laws. If you discover content in the Research Portal that you believe breaches copyright or violates any law, please contact openaccess@qub.ac.uk. 


\title{
Propionibacterium acnes and lipopolysaccharide induce the expression of antimicrobial peptides and proinflammatory cytokines/chemokines in human sebocytes
}

\author{
István Nagy ${ }^{\mathrm{a}, *}$, Andor Pivarcsi ${ }^{\mathrm{b}}$, Kornélia Kis ${ }^{\mathrm{a}}$, Andrea Koreck ${ }^{\mathrm{a}}$, László Bodai ${ }^{\mathrm{a}}$, Andrew \\ McDowell $^{\mathrm{c}}$, Holger Seltmann ${ }^{\mathrm{d}}$, Sheila Patrick ${ }^{\mathrm{c}}$, Christos C. Zouboulis ${ }^{\mathrm{d}, \mathrm{e}}$, Lajos Kemény ${ }^{\mathrm{a}, \mathrm{b}}$ \\ ${ }^{a}$ Department of Dermatology and Allergology, University of Szeged, Korányi fasor 6, 6720 Szeged, Hungary \\ ${ }^{\mathrm{b}}$ Dermatological Research Group of the Hungarian Academy of Sciences and the University of Szeged, Korányi fasor 6, 6720 Szeged, Hungary \\ ' School of Medicine and Dentistry, Queen's University, Grosvenor Road, Belfast BT12 6BN, UK \\ ${ }^{\mathrm{d}}$ Institute of Clinical Pharmacology and Toxicology, Charité Universitaetsmedizin Berlin, Campus Benjamin Franklin, Garystrasse 5, 14195 Berlin, Germany \\ ${ }^{\mathrm{e}}$ Departments of Dermatology and Immunology, Dessau Medical Centre, Auenweg 38, 06847 Dessau, Germany
}

Received 3 October 2005; accepted 11 April 2006

Available online 19 May 2006

\begin{abstract}
Acne is a common skin disorder of the pilosebaceous unit. In addition to genetic, hormonal and environmental factors, abnormal colonization by Propionibacterium acnes has been implicated in the occurrence of acne via the induction of inflammatory mediators. To gain more insight into the role that sebocytes play in the innate immune response of the skin, particularly in acne, we compared the antimicrobial peptide and proinflammatory cytokine/chemokine expression at mRNA and protein levels, as well as the viability and differentiation of SZ95 sebocytes in response to co-culture with representative isolates of $P$. acnes type IA and type IB as well as Escherichia coli-derived lipopolysaccharide (LPS). We found that, in vitro, $P$. acnes type IA and IB isolates and LPS induced human $\beta$-defensin- 2 and proinflammatory cytokine/chemokine expression, and influenced sebocyte viability and differentiation. Our results provide evidence that sebocytes are capable of producing proinflammatory cytokines/chemokines and antimicrobial peptides, which may have a role in acne pathogenesis. Furthermore, since $P$. acnes types IA and IB differentially affect both the differentiation and viability of sebocytes, our data demonstrate that different strains of $P$. acnes vary in their capacity to stimulate an inflammatory response within the pilosebaceous follicle.
\end{abstract}

(c) 2006 Elsevier SAS. All rights reserved.

Keywords: Acne; Sebocytes; Pilosebaceous unit; Inflammation; Innate immunity

\section{Introduction}

The human skin is densely populated with the resident microbiota, which is mainly composed of commensal micro-organisms, such as Propionibacterium acnes. This anaerobic Gram-positive bacterium is thought to play a central role in acne, a common skin disorder of the pilosebaceous unit characterized by abnormal ductal keratinization, increased

\footnotetext{
* Corresponding author. Tel.: +36 62 545799; fax: +36 62545954 . E-mail address: nagyi@mail.derma.szote.u-szeged.hu (I. Nagy).
}

sebum secretion, abnormalities of the skin microbiota and inflammation [1]. The lesions are localized in the acne-prone areas, where pilosebaceous follicles are most common. Pilosebaceous follicles consist of the hair follicle, hair shaft and sebaceous gland. The main function of the sebaceous glands, and the sebocytes within, is to produce and excrete sebum. Increased sebum secretion is associated with the development of acne lesions, since sebum serves as a nutrient source for $P$. acnes [2-4]. Other sebaceous gland functions, such as the production of proinflammatory lipids, cytokines, periglandular peptides and neuropeptides, are also associated with the pathophysiology of acne [4-6]. In addition, human sebocytes 
express functional receptors for corticotropin-releasing hormone [7,8], melanocortins [9], $\beta$-endorphin [10], vasoactive intestinal polypeptide and calcitonin gene-related peptide [11]. After the binding of the appropriate ligand, these receptors modulate the production of inflammatory cytokines, proliferation, differentiation, lipogenesis and androgen metabolism in sebocytes [12].

Inflammation in acne is associated, in part, with abnormal ductal colonization by $P$. acnes. The bacteria colonize sebum-rich follicles in the skin of the cheeks, nose, forehead, chest and back. Although the correlation between the severity of acne and the number of $P$. acnes organisms is controversial $[13,14]$, recent studies have shown that $P$. acnes triggers antimicrobial peptide and cytokine secretion of keratinocytes in vitro [15-17]. $P$. acnes is also able to induce the proliferation and differentiation of keratinocytes in vitro [16]. However, interpreting the results of previous studies investigating the role of $P$. acnes in the pathogenesis of acne is difficult, since potential differences amongst the recently discovered phylogenetic groups of $P$. acnes have not been taken into account $[18,19]$. Nucleotide sequencing of the recA housekeeping gene has demonstrated that the two serotypes of $P$. acnes, known as types I and II, represent phylogenetically distinct clusters or groups. Analysis of these sequences has also revealed a further cluster of isolates within the type I phylogenetic grouping, which have been designated type IB to distinguish them from other type I strains, designated type IA [18]. Type IB organisms display atypical monoclonal antibody (mAb) labelling characteristics with antibodies specific for types I and II when compared with other type IA strains, which is indicative of differences in the composition of the cell envelope. In addition, type IA and IB isolates also have distinct phenotypes with respect to the production of secreted components [19]. Recently, we observed that the expression profile of the antimicrobial peptide human $\beta$-defensin-2 (hBD2) and the proinflammatory chemokine CXC ligand-8 (CXCL8; previously referred to as interleukin [IL]-8) as well as the differentiation and proliferation of the keratinocytes after treatment with $P$. acnes, correlated with the RAPD (rapid amplification of polymorphic DNA) fingerprint of the bacteria [16]. Collectively, such data now challenge our current understanding of the pathogenic nature of $P$. acnes and raise the exciting possibility that specific virulent strains or groups of strains with a greater capacity to cause opportunistic infection may exist. This may explain, in part, the apparent controversy in the literature with respect to the role of $P$. acnes in acne.

In the skin, recognition of pathogen-associated molecular patterns (PAMPs) such as lipopolysaccharides (LPS), peptidoglycans (PGNs) or lipoteichoic acid (LTA) through patternrecognition receptors (PRRs) [20] leads to a series of signalling events resulting in the expression of proinflammatory cytokines/chemokines and antimicrobial peptides [21], which in turn mediates the elimination of pathogens [22]. The SZ95 human sebaceous gland cells express PRRs, such as Toll-like receptor (TLR)2, TLR4, TLR6 and CD14 [23,24]. Upon stimulation with LPS, PGN or LTA SZ95 sebocytes release cytokines in TLR- and CD14-dependent mechanisms, indicating that these receptors are indeed functional (Oeff et al., in preparation). To gain more insight into the role that sebocytes play in the innate immune response of the pilosebaceous unit, we compared the effect of isolates representing $P$. acnes types IA and IB, as well as Escherichia coli-derived LPS, on gene and protein expression of PRRs (TLR2 and TLR4), antimicrobial peptide (hBD2) and the proinflammatory cytokines/ chemokine (tumour necrosis factor(TNF)- $\alpha$, IL- $1 \alpha$ and CXCL8, respectively) by SZ95 sebocytes. Temporal changes in viability and differentiation of the SZ95 sebocytes were also determined.

\section{Materials and methods}

\subsection{P. acnes isolates and culture conditions}

Propionibacterium acnes isolates were cultured and stored as published in detail [16]. Briefly, P. acnes clinical isolates 889, 2005, 6609 and the reference strain ATCC11828 [16] were cultured on pre-reduced Columbia agar base (Oxoid, UK) supplemented with $5 \%(\mathrm{v} / \mathrm{v})$ bovine blood, vitamin $\mathrm{K}_{1}$ and haemin. Bacteria were grown under anaerobic conditions (anaerobic chamber; Bactron Sheldon Man, Oregon, USA) at $37{ }^{\circ} \mathrm{C}$. Single-cell colonies of $P$. acnes isolates were inoculated in broth cultures of brain heart infusion (BHI, pH 7.4; Oxoid), and the samples were incubated at $37{ }^{\circ} \mathrm{C}$ overnight to achieve logarithmic growth. Bacterial suspensions were then pelleted by centrifugation at $2600 \times g$ for $15 \mathrm{~min}$ and the supernatant discarded. After three washes in PBS-EDTA for $15 \mathrm{~min}$ each, the cells were harvested in $5 \mathrm{ml}$ PBSEDTA, and the cell number was estimated by measuring the optical density of the solution with a Cecil CE 2040 spectrophotometer (Cecil Instruments, Cambridge, UK) at $600 \mathrm{~nm}$. In some experiments, bacteria were heat treated $(30 \mathrm{~min}$, $\left.60{ }^{\circ} \mathrm{C}\right)$. After an additional centrifugation, the cells were harvested in complete sebocyte medium (see below) lacking antibiotic/antimycotic solution, aliquoted and stored at $-80{ }^{\circ} \mathrm{C}$ or $-20{ }^{\circ} \mathrm{C}$.

\subsection{Molecular, biochemical and immunological analyses of $\mathrm{P}$. acnes isolates}

\subsubsection{PCR amplification and sequencing of $\mathrm{P}$. acnes recA gene}

Preparations of bacterial genomic DNA and PCR amplifications were carried out as previously described [18]. PCR samples contained $1 \times$ PCR buffer, $200 \mu \mathrm{M}$ of each deoxynucleoside triphosphate (Amersham Biosciences, Little Chalfont, England), $200 \mu \mathrm{M}$ of the previously published recA oligonucleotide primers PAR-1 and PAR-2 [18], $1.5 \mathrm{mM} \mathrm{MgCl}_{2}$, 1.25 U Platinum Taq DNA polymerase (Invitrogen Life Technologies, Paisley, UK) and $2.5 \mu \mathrm{l}$ of bacterial lysate in a total volume of $25 \mu \mathrm{l}$. Samples were heated at $95{ }^{\circ} \mathrm{C}$ for $3 \mathrm{~min}$, followed by 35 cycles consisting of $1 \mathrm{~min}$ at $95^{\circ} \mathrm{C}, 30 \mathrm{~s}$ at $55^{\circ} \mathrm{C}$ and $1.5 \mathrm{~min}$ at $72{ }^{\circ} \mathrm{C}$. The PCR was completed with a final extension step at $72{ }^{\circ} \mathrm{C}$ for $10 \mathrm{~min}$. A negative water control was included in all experiments. PCR products were analysed as 
described before [18]. Sequencing reactions were performed using ABI PRISM ${ }^{\circledR}$ Ready Reaction Terminator Cycle Sequencing Kits (Perkin-Elmer Applied Biosystems, Foster City, CA) according to the manufacturer's instructions. Samples were analysed on an ABI PRISM 3100 DNA sequencer (Perkin-Elmer Applied Biosystems).

\subsubsection{Nucleotide sequence accession numbers}

recA nucleotide sequences were submitted to GenBank, and each was assigned an accession number as shown in Table 1.

\subsubsection{Phylogenetic analyses}

recA-based phylogenetic relationships between the different $P$. acnes isolates were determined as previously described [18]. Briefly, a phylogenetic tree showing $r e c A$ nucleotide sequence relationships was generated using Molecular Evolutionary Genetics Analysis software (MEGA; http://www.megasoftware.net) [25]. Using the CLUSTAL W algorithm [26], multiple sequence alignments were performed on the $r e c A$ sequences of our three isolates and reference strain, as well as previously published $P$. acnes recA sequences [18], which were selected to represent different phylogenetic clusters. A phylogenetic tree was constructed using the neighbour-joining method with the Jukes-Cantor-based algorithm. Sequence input order was randomised, and bootstrapping re-sampling statistics were performed using 100 data sets for each analysis.

\subsubsection{Fermentation analyses}

Fermentation analyses of $P$. acnes strains were performed as previously described [18]. Briefly, modified protease peptone yeast agar plates containing $40 \mathrm{mg}$ of bromocresol purple indicator (BDH, Poole, UK) per litre and 1\% (w/v) sorbitol, erythritol or ribose (Sigma, Poole, UK) were used. Bacteria were grown anaerobically, and a positive fermentation reaction was noted if the agar plates turned yellow due to acid production.

\subsubsection{Immunofluorescence microscopy of P. acnes strains}

Immunofluorescence microscopy (IFM) was carried out as described $[18,19]$. Briefly, bacterial cultures were grown on anaerobic blood agar, and a suspension of $10^{6} \mathrm{CFU}$ in $0.01 \mathrm{M}$ PBS was applied to multiwell slides, air dried and fixed in $100 \%$ methanol for $10 \mathrm{~min}$ at $-20{ }^{\circ} \mathrm{C}$. Undiluted mAbs QUBPa1 or QUBPa2, specific for P. acnes types IA and type II, respectively, [18] were added (30 $\mu \mathrm{l})$, and the samples were incubated at $37^{\circ} \mathrm{C}$ for $45 \mathrm{~min}$. After washing in PBS for 20 min, FITC-coupled goat anti-mouse immunoglobulin (Sigma) was added at 1:100 dilution in 0.1\% (v/v) Evans

Table 1

recA nucleotide sequence analyses of $P$. acnes isolates

\begin{tabular}{lll}
\hline Isolate & recA accession no. & $P$. acnes type by $r e c A$ sequencing \\
\hline ATCC11828 & DQ059328 & II \\
6609 & DQ059329 & IB \\
889 & DQ059330 & IA \\
2005 & DQ059331 & IA \\
\hline
\end{tabular}

Blue (Merck Sharp and Dome, Hoddesdon, UK) and counterstained for $45 \mathrm{~min}$ at $37{ }^{\circ} \mathrm{C}$. Slides were washed and mounted in glycerol-PBS containing an anti-photobleaching agent (Citifluor; Agar Scientific Ltd., Stansted, UK) and examined using a Leitz Dialux 20 fluorescence microscope.

\subsection{Culture and treatment of the SZ95 human sebaceous gland cell line}

The immortalized sebaceous gland cell (sebocyte) line SZ95 was used [27]. The cells were initially cultured in complete sebocyte medium (CSM) that contained Sebomed Basal Medium (Biochrom, Berlin, Germany), supplemented with $5 \mathrm{ng} / \mathrm{ml}$ human recombinant epidermal growth factor (Sigma), antibiotic/antimycotic solution (Sigma) and 10\% (v/v) heat-inactivated foetal bovine serum (FBS) (HyClone, USA), at $37{ }^{\circ} \mathrm{C}$ in a humidified atmosphere containing $5 \%$ $(\mathrm{v} / \mathrm{v}) \mathrm{CO}_{2}$ in air. At $24 \mathrm{~h}$ before treatment, the culture medium was switched to antibiotic/antimycotic-free medium. The cells were then treated with $5 \mu \mathrm{g} / \mathrm{ml}$ LPS purified from Escherichia coli 026:B6 (Sigma) or whole cells of P. acnes, at a $P$. acnes:sebocyte ratio of 100:1 as determined previously (data not shown). Treated sebocytes were cultured at $37{ }^{\circ} \mathrm{C}$ in an atmosphere of $5 \%(\mathrm{v} / \mathrm{v}) \mathrm{CO}_{2}$ in air for 3 and $24 \mathrm{~h}$ for mRNA expression, 12 and $36 \mathrm{~h}$ for protein expression, $24 \mathrm{~h}$ for proliferation, and 24 and $48 \mathrm{~h}$ for viability analyses before harvesting. For negative controls, SZ95 sebocytes were cultured with CSM lacking antibiotic/antimycotic solution (mock treatment). Following co-culture, the supernatants were collected and the cells harvested for mRNA expression, protein expression or viability analyses.

All tissue culture dishes, pipettes and the FBS used in our experiments were free of endotoxin contamination according to the manufacturers.

\subsection{Quantitative reverse transcriptase-polymerase chain reaction $(Q R T-P C R)$}

To quantify the relative abundance of selected mRNAs, realtime quantitative PCR was used following the protocol previously published [16]. Briefly, total RNA was isolated using TRIzol reagent (GibcoBRL, Eggstein, Germany) according to the manufacturer's instructions, and the RNA concentration was determined by the $A_{260}$ value of the sample. First-strand cDNA was synthesized from $1 \mu \mathrm{g}$ total RNA using the iScript ${ }^{\mathrm{TM}}$ cDNA Synthesis Kit (Bio-Rad, Hercules, CA) in a final volume of $20 \mu \mathrm{l}$. After transcription, amplification was carried out using iTaq DNA polymerase (Bio-Rad). The primer sets and TaqMan probes for $\mathrm{hBD} 2$ and $\mathrm{mACh}-\mathrm{R} \mathrm{m} 2$ were obtained from TaqMan Gene Expression Assays (Applied Biosystems, CA), while those for 18 S, CXCL8, IL-1 $\alpha$, TLR2, TLR4 and TNF- $\alpha$ have been specified elsewhere $[16,28,29]$. The amplification protocol contained one cycle of initial denaturation at $94{ }^{\circ} \mathrm{C}$ for $5 \mathrm{~min}$ followed by a varying number of cycles of denaturation at $94{ }^{\circ} \mathrm{C}$ for $1 \mathrm{~min}$, annealing at $55^{\circ} \mathrm{C}$ for $1 \mathrm{~min}$, extension at $72{ }^{\circ} \mathrm{C}$ for $2 \mathrm{~min}$ and one cycle of terminal extension at $72{ }^{\circ} \mathrm{C}$ for $10 \mathrm{~min}$. Each primer set gave a unique product. For 
controls, we used the reaction mixtures without the cDNA. Target gene expression was normalized between different samples based on the values of the expression of the internal positive control (18S rRNA).

\subsection{Immunofluorescent staining of SZ95 sebocytes}

The expression of $\mathrm{hBD} 2$ protein was determined by indirect immunohistochemistry as described in detail [16]. Briefly, cell suspensions of $1 \times 10^{5}$ cells in $50 \mu \mathrm{l}$ PBS were cytospun, fixed in freshly prepared $4 \%(\mathrm{w} / \mathrm{v})$ paraformaldehyde in PBS, permeabilized with PBS containing $1 \%$ (v/v) Triton-X (Sigma) and blocked in PBS containing 1\% (w/v) BSA. The antibodies used in the experiments were a polyclonal rabbit anti-hBD2 (1:333; a kind gift of Dr. T. Ganz, UCLA, CA) and FITCcoupled goat anti-rabbit immunoglobulin (1:90; Sigma). The samples were mounted in mounting medium $(100 \mathrm{mM}$ Tris $\mathrm{pH} 8.5 ; 25 \%$ glycerol) and analysed using epifluorescent illumination of the Axioplan microscope (Opton, Germany). Images were recorded with an RT KE camera and SPOT RT v4.0 software (Diagnostic Instruments). In all cases time-matched negative controls consisted of preincubation with PBS or substitution of the primary antibody with pre-immune serum.

\subsection{ELISA}

Human CXCL8 and TNF- $\alpha$ proteins were measured from the supernatants of SZ95 sebocytes using the Quantikine human CXCL8 immunoassay kit (R\&D Systems Inc., Minneapolis, MN), Quantikine hTNF- $\alpha$ /TNFSF1A Immunoassay kit (R\&D Systems) and Biosource (Nivelles, Belgium) hTNF$\alpha$ ELISA kit, according to the manufacturers' protocols. Serial dilutions of recombinant human CXCL8 and TNF- $\alpha$ were used for standard curves. The optical density of the wells was determined using a microtitre plate reader (Multiscan v3.0, Labsystems) set to a wavelength of $450 \mathrm{~nm}$, with a wavelength correction set to $540 \mathrm{~nm}$ for Quantikine kits and $620 \mathrm{~nm}$ for Biosource kits.

\subsection{Cycloheximide treatment}

To determine the role of de novo protein synthesis, cycloheximide (CHX) was used as protein synthesis inhibitor. CHX (Sigma) was dissolved either in $0.01 \%$ dimethyl sulfoxide (DMSO) or in PBS. Sebocytes were mock, LPS or P. acnes treated in the presence of 2,10 or $20 \mu \mathrm{g} / \mathrm{ml} \mathrm{CHX}$ in parallel with control samples that were treated with the vehicle only. After 3 or $24 \mathrm{~h}$, samples were collected, and mRNA or protein levels were determined as described above.

\subsection{Measurement of cell viability by methylthiazoletetrazolium (MTT) assay}

The MTT assay, based on the enzymatic reduction of the tetrazolium salt MTT in viable/metabolically active cells, was used to monitor cell viability. SZ95 sebocytes were seeded in each well of a 96-well plate at a density of $3 \times 10^{3}$ cells/well.
The treatment of the SZ95 sebocytes was performed using LPS, the $P$. acnes clinical isolates 889 or 6609 or with medium alone which served as a control. At 3, 24 and $48 \mathrm{~h}$ post treatment, the supernatant was replaced with RPMI and the assay was performed as published in detail [16].

\subsection{Data presentation}

To evaluate cell viability, the average absorbance due to cell growth in treated wells was expressed as a percentage of the average absorbance due to cell growth in control wells (regarded as 100\%). Each plate was evaluated at one time point, and at least 10 wells comprised one group. Results due to QRT-PCR were expressed as fold increases over the time-matched mock-treated control values. All the data were presented as mean \pm standard deviation for at least three experiments. Data were compared using one-way analysis of variance (ANOVA) followed by Dunnett's post hoc test to determine statistical differences after multiple comparisons (SPSS, SPSS Inc., Chicago, IL). A probability value of less than 0.05 was considered significant.

\section{Results}

\subsection{Genetic and phenotypic differences between P. acnes isolates}

The phylogenetic cluster of our $P$. acnes isolates (Table 1) was determined by sequencing of the rec $A$ gene. Isolates 889 and 2005 clustered with type IA strains, isolate 6609 clustered with type IB, while the strain ATCC11828 clustered with type II organisms (Fig. 1). Additional molecular analyses of the isolates by RAPD fingerprinting [16] confirmed the phylogenetic groupings of type I or II as determined by recA sequencing.

Fermentation analyses with sorbitol, erythritol and ribose substrates indicated that isolates 6609,889 and 2005 had the biotype profile $\mathrm{B} 1$, which is in keeping with their status as type I strains. ATCC11828 displayed the biotype profile B2, characteristic of type II organisms (Table 2). The mAb QUBPa3 [30], which reacts with all $P$. acnes isolates examined to date $(n>100)$, reacted with all four isolates used in the current study by IFM (data not shown). Type IA isolates 889 and 2005 reacted with the previously described type IAspecific mAb QUBPa1 [18], but showed no reaction with the type II-specific mAb QUBPa2. Isolate 6609 exhibited $\mathrm{mAb}$ labelling characteristic of type IB strains [18]. No reaction was observed with mAb QUBPa1, but weak labelling with QUBPa2 was detected. Labelled cells showed significantly reduced fluorescence intensity and an uncharacteristic reduction in the proportion of the bacterial population labelled. Strain ATCC11828 displayed atypical mAb labelling and reacted with both the type IA- and II-specific antibodies.

Pathogenic strain 889 was isolated from a male patient with $P$. acnes-induced endocarditis. Isolate 6609 was recovered from the skin sample of a woman with no recorded acne history, and is thus considered a non-pathogenic strain. These two 


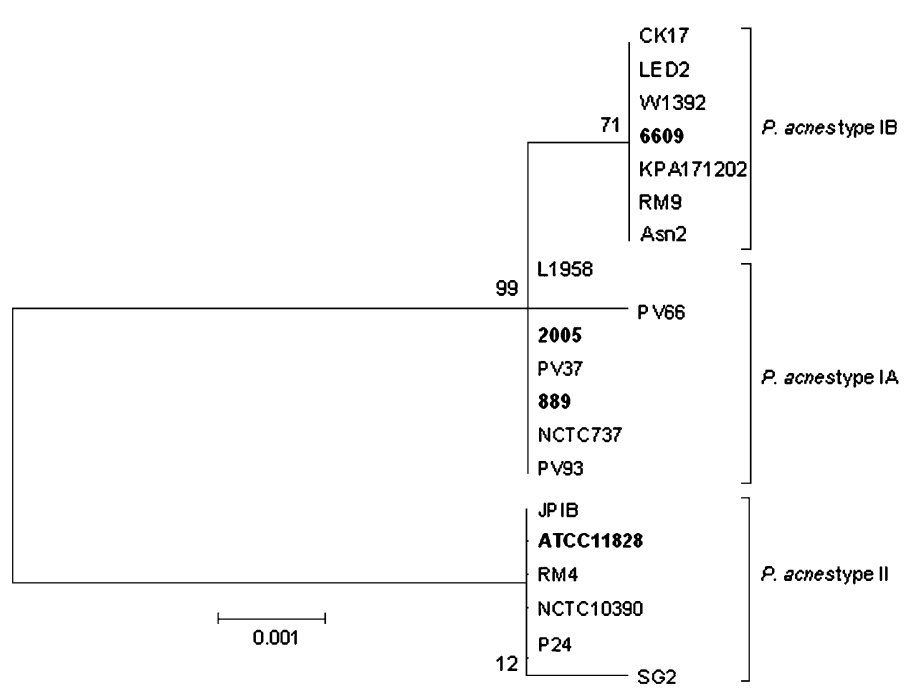

Fig. 1. Unrooted phylogenetic tree of $P$. acnes based on the complete $r e c A$ gene sequence. Multiple sequence alignments were performed on the $r e c A$ sequences from $P$. acnes reference strain ATCC11828 and clinical isolates 6609, 889 and 2005, as well as published recA sequences from other $P$. acnes strains selected to represent different phylogenetic clusters. Bootstrapping resampling statistics were applied to the tree (100 data sets), and the bootstrap values are shown at different nodes on the tree. The type status for the different strains is shown on the right.

clinical isolates, representative of type IA and IB respectively, were used in subsequent studies.

\subsection{P. acnes strains and LPS induce the expression of the antimicrobial peptide $h B D 2$ in SZ95 sebocytes}

Different strains of $P$. acnes, as well as other Gram-positive and also Gram-negative bacteria, induce the expression of hBD2 in keratinocytes [16,31]. To further investigate the effect of $P$. acnes types IA, IB and II, as well as E. coli LPS on the $\mathrm{hBD} 2$ expression of different cell types of the pilosebaceous unit, we determined whether exposure of SZ95 sebocytes to $P$. acnes type IA, IB or LPS had any effect on the expression of hBD2. For this, SZ95 sebocytes were mock, P. acnes type IA, IB or LPS treated, and the effect of the treatments on the gene expression of SZ95 sebocytes was determined using QRT-PCR. As shown in Fig. 2, the exposure of SZ95 sebocytes to $P$. acnes types IA, IB and LPS significantly $(P<0.05)$ induced the mRNA expression of $\mathrm{hBD} 2$, at $24 \mathrm{~h}$ post treatment, when compared to time-matched mock-treated control values. In contrast, type II isolate had no effect on hBD2 expression (data not shown). In order to test whether the increased amount of hBD2 mRNA was due to an increase

Table 2

Comparison of $P$. acnes isolates by fermentation and immunofluorescence microscopy analyses

\begin{tabular}{|c|c|c|c|c|c|c|c|}
\hline \multirow[t]{2}{*}{ Isolate } & \multicolumn{4}{|c|}{ Fermentation results } & \multicolumn{3}{|c|}{ IFM results } \\
\hline & Sorbitol & Erythritol & Ribose & Biotype & QUBPa1 & QUBPa2 & $\begin{array}{l}\text { Type by } \\
\text { mAb } \\
\text { labelling }\end{array}$ \\
\hline ATCC11828 & - & + & + & B2 & + & + & Atypical \\
\hline 6609 & + & + & + & B1 & - & $\mathrm{W}+$ & Atypical \\
\hline 889 & + & + & + & B1 & + & - & IA \\
\hline 2005 & + & + & + & B1 & + & - & IA \\
\hline
\end{tabular}

in protein synthesis, cells were pretreated with the protein synthesis inhibitor cycloheximide (CHX). CHX pretreatment had no effect on the $P$. acnes type IA- and IB-induced hBD2 mRNA levels (data not shown), indicating that it is not dependent on de novo protein synthesis. Interestingly, CHX further upregulated LPS-induced hBD2 mRNA levels at $24 \mathrm{~h}$ post treatment (1.5-fold upregulation; $P=0.005$ ).

Immunofluorescent staining of mock-, LPS- and P. acnestreated SZ95 sebocytes using a specific antibody directed against hBD2 confirmed the results obtained at the mRNA level (Fig. 3). At 12 and $36 \mathrm{~h}$ post treatment, hBD2-expressing cells were detected in $P$. acnes type IA-, IB- and LPS-treated SZ95 sebocytes (data not shown and Fig. 3). However, the labelled cells at $12 \mathrm{~h}$ post treatment showed reduced fluorescent intensity compared to the fluorescent intensity of the labelled cells at $36 \mathrm{~h}$ post treatment, indicative of an upregulated $\mathrm{hBD} 2$ protein expression.

\subsection{P. acnes type IA and IB and LPS induce the expression of the proinflammatory chemokine CXCL8 and cytokine TNF- $\alpha$, but not IL-1 $\alpha$, in SZ95 sebocytes}

Based on reports that LPS and $P$. acnes induce proinflammatory cytokine/chemokine expression in keratinocytes $[15,16,28]$, we conducted a series of time-course experiments, using QRT-PCR, to investigate SZ95 sebocyte expression of proinflammatory cytokines (TNF- $\alpha$ and IL- $1 \alpha$ ) and the chemokine CXCL8 upon treatment with $P$. acnes type IA, IB or LPS. P. acnes type IA, IB and LPS significantly upregulated the expression of both CXCL8 and TNF- $\alpha$ mRNA when compared to time-matched mock-treated control values (Fig. 2). Interestingly, the time taken to induce CXCL8 and TNF$\alpha$ mRNA levels by $P$. acnes types IA and IB was significantly longer compared to induction with LPS. LPS also induced IL-1 $\alpha$ mRNA expression at $3 \mathrm{~h}$ post treatment; however, 


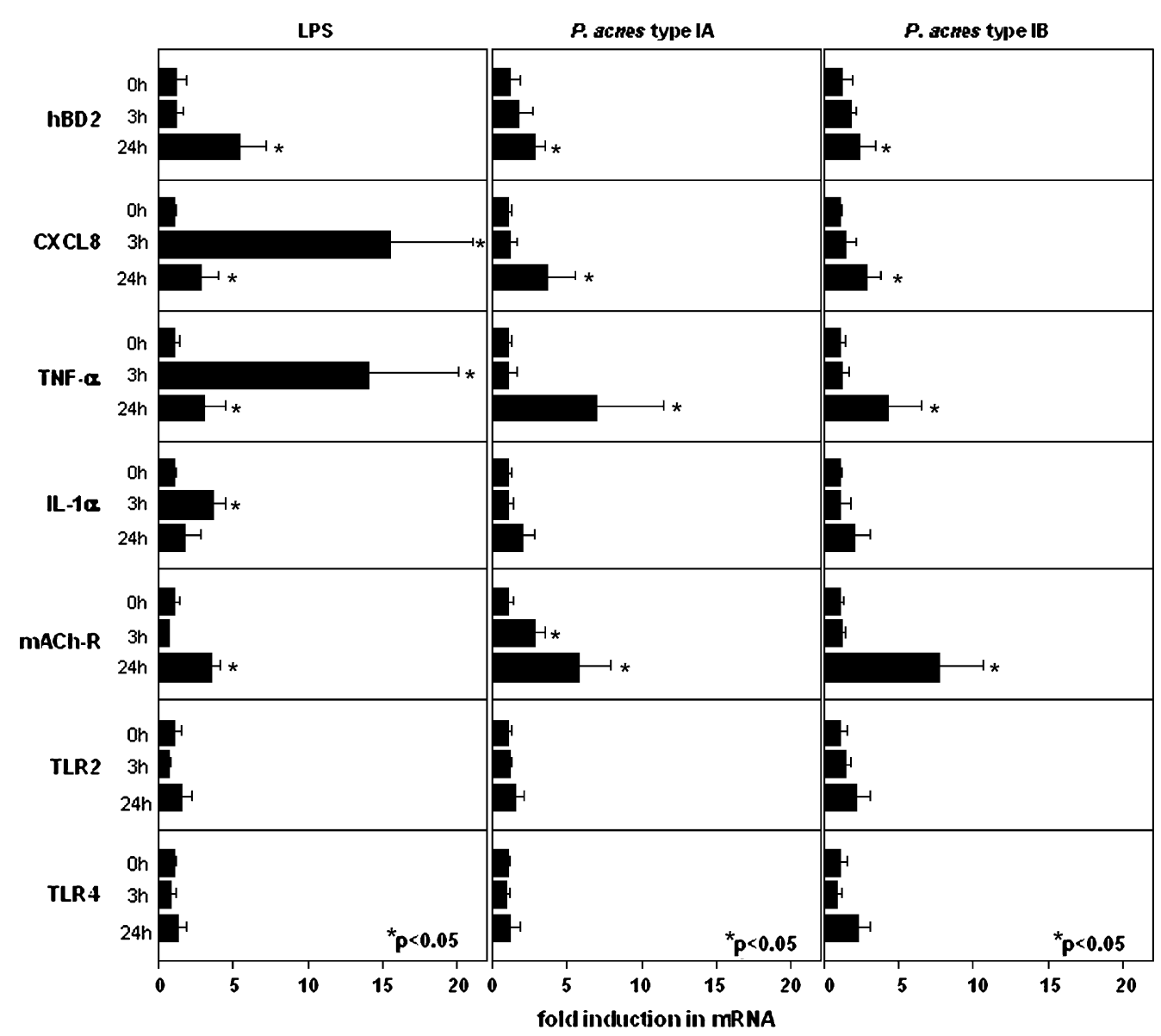

Fig. 2. Treatment with LPS, P. acnes type IA or type IB isolates differentially induces the gene expression of SZ95 sebocytes. Temporal analyses of gene expression of antimicrobial peptide (hBD2), cytokines (TNF- $\alpha$, IL-1 $\alpha$ ) chemokine (CXCL8), muscarinic acetylcholine receptor (mACh-R m2) and Toll-like receptors (TLR2 and TLR4) in SZ95 sebocytes treated with LPS, P. acnes type IA (clinical isolate 889) or P. acnes type IB (clinical isolate 6609) using QRT-PCR. The levels of mRNA were normalized to the 18S rRNA level and are presented as fold increase over the time-matched mock-treated control values. Results are mean \pm S.D. of at least three independent experiments.

P. acnes types IA and IB had no effect on IL-1 $\alpha$ mRNA levels (Fig. 2).

To analyse whether protein secretion was also induced, we examined the supernatants of mock-, LPS- and P. acnestreated SZ95 sebocytes for CXCL8 and TNF- $\alpha$ by ELISA. Treatment of SZ95 sebocytes with LPS resulted in a significant increase in the secretion of CXCL8 protein at 24 and $36 \mathrm{~h}$ post treatment $(P<0.001$; Fig. 4). Co-culture of SZ95 sebocytes with $P$. acnes types IA and IB had no effect on CXCL8 protein expression at $24 \mathrm{~h}$ post treatment, but did induce significant CXCL8 protein secretion at $36 \mathrm{~h}$ post treatment $(P<0.05$; Fig. 4). Furthermore, blockade of protein synthesis with CHX did not prevent the cells from secreting CXCL8, on the contrary, CHX treatment elevated the amount of LPS-induced CXCL8 secretion at $24 \mathrm{~h}$ (data not shown). Since $P$. acnes type IA, IB and LPS also upregulated the mRNA level of TNF- $\alpha$, we sought to measure the secreted TNF- $\alpha$ protein as well. For this, two commercially different TNF- $\alpha$ ELISA kits were used, in which the minimal detectable TNF- $\alpha$ concentration was 1.6 and $3 \mathrm{pg} / \mathrm{ml}$. No secreted TNF- $\alpha$ protein was detected in the supernatants from any of the treatments (data not shown).

\subsection{P. acnes and LPS have no effect on the TLR2 and TLR4 expression of SZ95 sebocytes}

As reported previously, SZ95 sebocytes express functional TLR2 and TLR4 $[23,24]$ (Oeff et al., in preparation). To determine the effect of treatment with $P$. acnes types IA, IB or LPS on TLR2 and TLR4 mRNA expression, QRT-PCR analyses were performed. As shown in Fig. 2, neither LPS nor P. acnes type IA or IB isolates modulated the expression of TLR2 or TLR4 in SZ95 sebocytes.

\subsection{The differentiation of SZ95 sebocytes is modulated by P. acnes and LPS}

Recently, an upregulation in the expression of the $\mathrm{m} 2$ subunit of the muscarinic acetylcholine receptor (mACh-R) was demonstrated in mature sebocytes as compared with the undifferentiated sebocytes [32]. To determine whether treatment with $P$. acnes types IA, IB or LPS modulated the differentiation of SZ95 sebocytes; we analysed the expression of the $\mathrm{m} 2$ $\mathrm{ACh}-\mathrm{R}$ gene in sebocytes before and at 3 and $24 \mathrm{~h}$ post treatment. Results of real-time RT-PCR analyses revealed that 

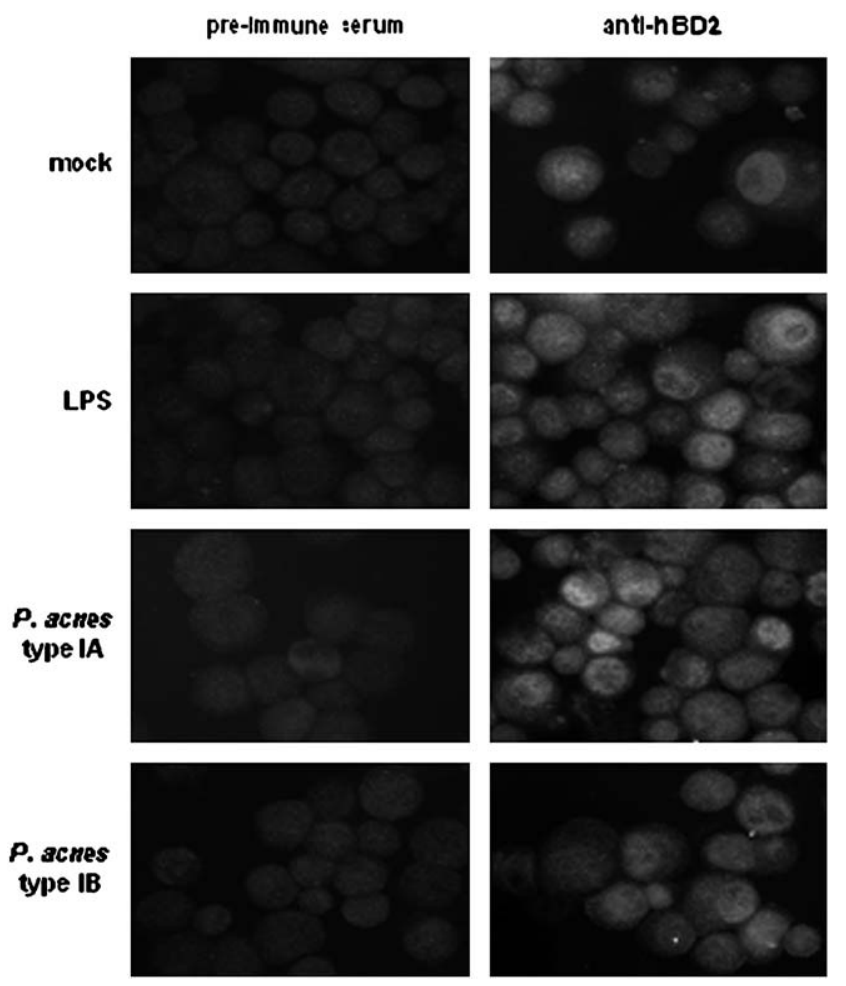

Fig. 3. Expression of hBD2 protein in treated SZ95 sebocytes. SZ95 sebocytes were mock, LPS, $P$. acnes type IA or $P$. acnes type IB treated for $36 \mathrm{~h}$, and the production of hBD2 was detected by immunofluorescence microscopy analyses. Magnification: $\times 400$.

$P$. acnes types IA and IB and LPS had a significant stimulatory effect on $\mathrm{m} 2$ ACh-R mRNA expression at $24 \mathrm{~h}$ post treatment $(P<0.05$; Fig. 2). However, in contrast to LPS and $P$. acnes type IB, $P$. acnes type IA induced the gene expression of $\mathrm{m} 2 \mathrm{ACh}-\mathrm{R}$ as soon as $3 \mathrm{~h}$ post treatment (Fig. 2).

\subsection{P. acnes types IA and IB isolates differentially modulate the viability of SZ95 sebocytes}

To investigate the temporal effects of $P$. acnes and LPS treatment on the viability of SZ95 sebocytes, MTT assays were performed. This assay reflects cellular growth and survival [33]. Results of these assays revealed that treatments with $P$. acnes types IA and IB but not LPS, significantly increased the viability of SZ95 sebocytes (Fig. 5), although the kinetics of the effects were different. Treatment with both $P$. acnes isolates significantly increased the number of viable cells as compared with untreated controls after 24 and $48 \mathrm{~h}$ of co-culture $(P<0.04$; Fig. 5). However, the ability of $P$. acnes type IB to induce sebocyte growth at both time points was significantly higher $(P<0.05)$ compared to $P$. acnes type IA (Fig. 5).

\section{Discussion}

Acne is a common, chronic inflammatory disease of the pilosebaceous follicle, affecting about 70-80\% of adolescents and young adults [5,34]. Although acne is not an infectious disease, the role of $P$. acnes, a Gram-positive bacterium that colonizes the pilosebaceous unit, is outlined by several data. Increased cellular and humoral immunity against $P$. acnes has been detected in many patients with acne $[1,35]$. Furthermore, in the affected follicles, the production of sebum from the sebaceous gland, as well as the clinical improvement of acne, correlates with the number of $P$. acnes organisms $[14,36]$. Recent data provide further support for genetic and phenotypic diversity within the $P$. acnes species. Jappe and colleagues demonstrated that a laboratory strain and fresh clinical isolates of $P$. acnes differed in their ability to stimulate naïve and adult lymphocytes [37]. Also, in our previous work, we demonstrated that the capacity of different $P$. acnes isolates to stimulate antimicrobial peptide expression in keratinocytes correlated with their RAPD genotype [16]. Using recently described methods for phylogenetic and biochemical analysis of $P$. acnes $[18,19]$, we now present evidence that the strains included in this study, as well as previous studies [16], represent phylogenetically and functionally distinct groups. On the basis of recA sequencing, the clinical isolates 889 and 2005 fell within the recently described type IA cluster, whereas isolate 6609 belonged within the type IB cluster. Immunofluorescence labelling with specific mAbs confirmed these results. Although ATCC11828 clustered with other type II isolates upon $\operatorname{rec} A$ sequence analysis, it did exhibit

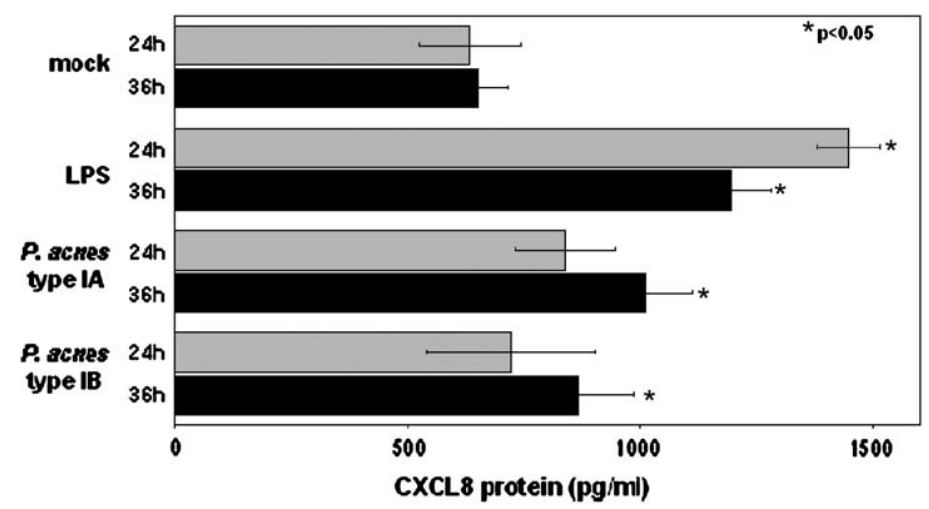

Fig. 4. LPS and P. acnes types differentially affect the CXCL8 protein secretion of SZ95 sebocytes. Cultured SZ95 sebocytes were mock, LPS or P. acnes type IA or type IB treated, and the amount of CXCL8 protein in the supernatant medium was measured with human CXCL8 ELISA. 


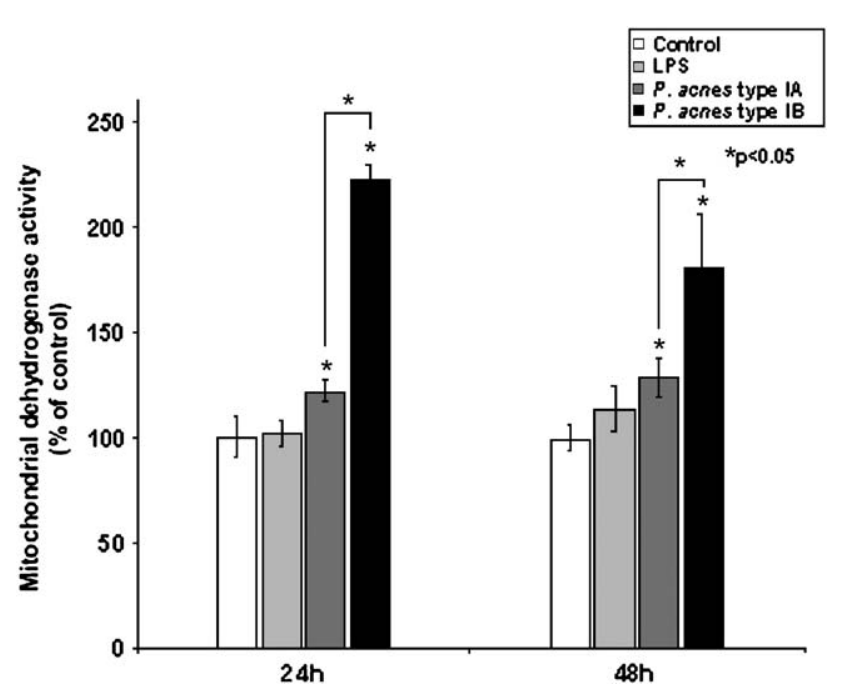

Fig. 5. P. acnes type IA and type IB isolates differentially influence the viability of SZ95 sebocytes. Cultured SZ95 sebocytes were mock-, LPS- or P. acnes type IA isolate- or type IB isolate-treated, and the temporal effect of the treatments on the viability of sebocytes was determined by MTT assay.

an atypical labelling pattern with the mAbs. To date, this labelling pattern has only been observed in 2 of 38 type II P. acnes isolates examined (unpublished). Interestingly, Johnson and Cummins, who designated ATCC11828 as type II, did not record the results obtained for the reaction of this isolate with antisera used to type other isolates in their study [38]. In addition, the percentage DNA homology of ATCC11828 with a reference type II isolate is only $90 \%$, whereas it is $94 \%$ with a reference type I [38].

A variety of host defence mechanisms control the resident microbiota in human skin. Sebocytes are proposed to participate in several pathophysiologic processes, especially in the pathogenesis of acne [1,4,6]. Little is known, however, about the exact role that sebocytes play in the regulation of immune function and inflammation following normal and increased $P$. acnes colonization. The immortalized human facial sebaceous gland cell line SZ95 exhibits properties of normal sebocytes, such as expression of characteristic sebaceous gland proteins and development of oil red $\mathrm{O}$ stain-/Nile red stain-positive lipid droplets in their cytoplasm [27]. It, therefore, represents a suitable model for the $P$. acnes-induced response within the pilosebaceous unit. The work described herein provides evidence that $P$. acnes and LPS induce the expression of antimicrobial peptides and proinflammatory cytokines/chemokine in, as well as differentiation and viability of, the SZ95 sebocyte line. In addition, the contribution of isolates representing $P$. acnes type IA and IB clusters to these processes is different, since they differentially affect the differentiation and viability of the sebocyte line.

The pathogenesis of acne is complex and involves genetic, hormonal and environmental aspects $[1,2,4]$. Macrophages, T cells, keratinocytes and sebocytes are all likely to be involved in the formation of acne lesions $[6,15,16,39,40]$. Within the sebaceous follicle, abnormal viability, proliferation and differentiation of keratinocytes and sebocytes results in increased sebum production. Several factors are believed to be involved in the initiation of sebocyte and keratinocyte proliferation and differentiation within the sebaceous follicle [4]. In our model, $P$. acnes type IA and type IB differentially affected the viability and differentiation of sebocytes. Our recent studies have highlighted that differences exist between type IA and type IB strains in the production of cAMP factors as well as cell wall antigen composition $[18,19]$. Thus, the ability of type IA and type IB isolates to differentially modulate the viability and differentiation of sebocytes (this study) and keratinocytes [16] are pointing out the role of $P$. acnes cell wall antigens and/or secreted proteins in acne development.

Several data suggest a complex role for the small antimicrobial peptide hBD2 in cutaneous host defence. Besides having a direct microbicidal effect against various micro-organisms [41-43], hBD2 acts as a chemoattractant for immature dendritic cells and neutrophils and also induces the migration of memory T cells $[44,45]$. Our results showing that LPS and isolates of $P$. acnes types IA and IB, but not type II (data not shown), upregulated hBD2 expression in sebocytes are in good agreement with previous results demonstrating upregulation of hBD2 in acne lesions [46,47] and the expression of hBD2 by $P$. acnes type IA-treated keratinocytes [16]. Furthermore, the expression of hBD2 by sebocytes is not dependent on de novo protein synthesis. Interestingly, recombinant hBD2 up to the concentrations tested has no direct antimicrobial effect on $P$. acnes [16]. Even though the concentration of hBD2 on the skin surface is not known, our findings may provide an attractive explanation for the role of hBD2 in acne development: it may contribute to the recruitment of neutrophils and $\mathrm{T}$ cells to the sites of infection, hence initialising inflammatory events.

Proinflammatory cytokines/chemokines secreted by various cell types play a fundamental role in attracting neutrophils and leukocytes to the place of skin infection [48]. P. acnes is able to induce the secretion of the chemokine CXCL8 in monocytes and keratinocytes [16,49]. In SZ95 sebocytes, LPS and $P$. acnes types IA and IB isolates upregulated CXCL8 expression and secretion without de novo protein synthesis, indicating that CXCL8 is stored in vesicles, as in keratinocytes. Interestingly, the LPS-induced increase in CXCL8 secretion was further upregulated by $\mathrm{CHX}$. A similar stimulatory effect of CHX was reported in other cellular models as well, and it is suggested that the observed effects are due to the inhibition of de novo protein synthesis of $\mathrm{I} \kappa \mathrm{B} \alpha$, the protein that suppresses the activation of NF- $\kappa \mathrm{B}$ [50]. It is noteworthy, however, that the effects of LPS and the various $P$. acnes types on the expression of CXCL8 are slightly different. Upregulation of the CXCL8 gene and protein expression after LPS challenge was found to occur at 3 and $24 \mathrm{~h}$ post treatment, respectively. In contrast, treatment with $P$. acnes types IA and IB resulted in CXCL8 gene and protein expression at 24 and $36 \mathrm{~h}$ post treatment, respectively. Our data are in good agreement with previous results that have demonstrated sebocytes to constitutively express CXCL8 without any external influence [8,9]. Since CXCL8 promotes the proliferation of keratinocytes [51] through the CXCL8 receptor [52], it is likely that the 
elevated level of CXCL8 protein in acne contributes to the hyperproliferation of ductal keratinocytes.

Upon treatment with $P$. acnes, keratinocytes secrete various cytokines, such as TNF- $\alpha$, IL- $1 \alpha$ and granulocyte/macrophage colony stimulation factor (GM-CSF) [15]. Our results demonstrating that viable, exponential-phase $P$. acnes fails to induce IL-1 $\alpha$ gene expression in SZ95 sebocytes confirm previous reports. Collectively, these data emphasize the role of the growth phase of $P$. acnes in the ability of the bacteria to stimulate cytokine secretion. In keratinocytes, only stationary- but not exponential-phase live bacteria induced IL- $1 \alpha$ secretion $[15,16]$. In contrast, both stationary- and exponential-phase live bacteria induced TNF- $\alpha$ and GM-CSF secretion [15]. In the current study, LPS and isolates of $P$. acnes types IA and IB, but not type II (data not shown), upregulated TNF- $\alpha$ gene expression in SZ95 sebocytes. Interestingly, despite the upregulated gene expression, we could not detect TNF- $\alpha$ protein in cell culture supernatants. The TNF- $\alpha$ protein plays an important role in the initiation and persistence of inflammation in a variety of skin disorders. Since persistent inflammation is a disadvantage for the host, and the resident microbe, silencing mechanisms such as posttranscriptional modification may regulate TNF- $\alpha$ production. Accumulating evidence supports the role of small interfering (si)RNAs and micro (mi)RNAs in posttranscriptional silencing mechanisms [53]. Currently, the possible role of siRNAs and miRNAs in posttranscriptional silencing of TNF- $\alpha$ following $P$. acnes treatment is under investigation.

Microbes and microbial-derived compounds induce the expression of proinflammatory cytokines/chemokines and antimicrobial peptides in keratinocytes through TLR-mediated signalling pathways [48,54]. Furthermore, $P$. acnes will induce the release of IL-6 from wild-type, TLR6 ${ }^{-1-}$ and TLR1 $1^{-1-}$ mouse-derived macrophages, but not from TLR2 ${ }^{-/-}$mousederived macrophages [39]. Since pilosebaceous follicles are surrounded with TLR2-expressing macrophages in acne lesions [39], it is likely these cells also recognize $P$. acnes. Isolates of $P$. acnes also induce cytokine production of monocytes via TLR2 [39] and antimicrobial peptide and chemokine production of keratinocytes via both TLR2 and TLR4 [16]. The well-known anti-inflammatory drug all-trans retinoic acid, used for the treatment of acne, downregulates the expression of TLR2 in primary human monocytes [55,56]; however, this is associated with increased rates of skin colonization and infection [24], which suggests that TLR2 upregulation is likely to have a protective effect on human skin and is required for a permanent low-activation level of innate immunity that quickly defends the skin from acute attack by different pathogens [24]. This supports the hypothesis that acne has a genuine inflammatory aetiology and suggests a delayed contribution by $P$. acnes [4,5].

Our findings demonstrate that sebocytes contribute to the innate immune response of the skin by producing antimicrobial peptides and proinflammatory cytokines/chemokines. It is noteworthy, however, that LPS and P. acnes isolates exhibited different kinetics of induction, with respect to CXCL8, TNF- $\alpha$ and IL- $1 \alpha$ expression. It is reasonable to propose that differences in composition and concentration of
PRRs may influence the response of the host cells. In sebocytes, similarly to other immune cells, microbes and microbial components induce cytokine release via TLR- and CD14dependent mechanisms (Oeff et al., in preparation). Even though $P$. acnes derived ligands of TLRs are yet to be identified, it is likely that $P$. acnes proteins such as GroEL, DnaK [57] or lipoglycans [58] may act as such ligands [48]. In addition, the genome sequence of $P$. acnes encodes many other factors that may have immunomodulatory potential [59]. The first comparison of the genome sequences of type IA and type IB strains identified varying numbers of base-pair and amino acid differences for a selection of secreted and surface-associated proteins, although there was $100 \%$ identity in the amino acid sequence of GroEL [19]. In our experimental model, neither LPS nor $P$. acnes types IA, IB or type II (data not shown) had any direct effect on the expression of either TLR2 or TLR4. These findings raise an attractive explanation for the role of sebocytes in skin inflammation: (1) sebocytes are sensing microbes via constitutively expressed PRRs, and (2) signal transduction pathways are indeed functional, as (3) effector proteins are secreted. However, as the expression of PRRs is not upregulated, it is most likely that upon microbial induction, the capacity of sebocytes to attract effector cells is limited.

McLorinan and colleagues have recently sampled different sites of the healthy skin and have shown that the areas most heavily colonized by $P$. acnes are the neck and the forehead, where $P$. acnes was most frequently detected in mixed type I and type II populations [60]. Although only a limited number of studies concerning the distribution of $P$. acnes types and the severity of clinical conditions, including acne, exists, it is important to note that isolates recovered from patients with acne were predominantly type IA ([18] and unpublished data). In vitro data, demonstrating that type I isolates exhibited stronger stimulatory effect on the antimicrobial peptide and proinflammatory cytokines/chemokine expression as well as differentiation and proliferation of both keratinocytes [16] and sebocytes (this study) are, indeed, reflecting the in vivo situation. These data are indicative of equilibrium between types of $P$. acnes within a single follicle, where changes of physiological conditions, such as increased sebum secretion, may lead to an imbalance between types and to the overgrowth of type I strains.

It has been recently proposed that future compounds developed to treat acne should be able to reduce proinflammatory lipids in sebum, downregulate proinflammatory signals in the pilosebaceous unit and reduce sebum production $[6,61]$. This is supported by results of our group [24], which provide further evidence for the hypothesis that $P$. acnes is involved in the formation of acne inflammation, but does not act as an infectious agent. Further support comes from the observations that acne responds to sub-antimicrobial-dose tetracyclines and possibly to the para-antibiotic activity of antibacterial agents despite its resistance to standard antibiotic agents [62-64].

In summary, we have demonstrated that the treatment of SZ95 sebocytes with P. acnes or LPS triggers the expression of antimicrobial peptides and proinflammatory cytokines/chemokines. 
By modulating both CXCL8 and hBD2 expression, P. acnes may contribute to the recruitment of inflammatory infiltrate, thus resulting in the modulation of inflammatory responses within the pilosebaceous unit. At the same time, P. acnes could contribute to proliferation and differentiation of sebocytes, which may also result in changes in sebum excretion. Taken together, our findings corroborate the hypothesis that sebocytes play an important role in the initiation and exacerbation of inflammation within the pilosebaceous unit. Furthermore, the observation that different isolates of $P$. acnes may differ in their contribution to acne pathogenesis raises the exciting possibility that we may be able to identify $P$. acnes strains, or groups of strains, with a greater potential to be involved in this condition, as well as putative virulence factors associated with such organisms.

\section{Acknowledgements}

We thank Dr. Tomas Ganz, University of California, Los Angeles, for providing the anti-hBD2 antibody and Dr. Edit Urbán, Department of Microbiology, University of Szeged, Hungary, for providing clinical isolates of $P$. acnes. We are grateful to Andrea Bajkán Tanácsné, Hilda Polyánka and Gisli Einnarsson for technical support. The work in the Department of Dermatology and Allergology, University of Szeged, Hungary was supported by OTKA (grants T 042738 and TS 044826), NKFP (grant 1A/0012/2002) and a William J. Cunliffe Scientific Award. The work in the School of Medicine and Dentistry, Queen's University, Belfast was supported by a Health and Personal Social Services Northern Ireland Programme Grant.

\section{References}

[1] A. Koreck, A. Pivarcsi, A. Dobozy, L. Kemeny, The role of innate immunity in the pathogenesis of acne, Dermatology 206 (2003) 96-105.

[2] M. Toyoda, M. Morohashi, Pathogenesis of acne, Med. Electron. Microsc. 34 (2001) 29-40.

[3] D. Thiboutot, Regulation of human sebaceous glands, J. Invest. Dermatol. 123 (2004) 1-12.

[4] C.C. Zouboulis, A. Eady, M. Philpott, L.A. Goldsmith, C. Orfanos, W.C. Cunliffe, R. Rosenfield, What is the pathogenesis of acne? Exp. Dermatol. 14 (2005) 143-152.

[5] C.C. Zouboulis, Is acne vulgaris a genuine inflammatory disease? Dermatology 203 (2001) 277-279.

[6] C.C. Zouboulis, Acne and sebaceous gland function, Clin. Dermatol. 22 (2004) 360-366.

[7] A. Slominski, G. Ermak, J. Hwang, A. Chakroborty, J. Mazurkiewitz, M. Mihm, Proopiomelanocortin, corticotrophin releasing hormone and cortocotrophin releasing hormone receptor genes are expressed in human skin, FEBS Lett. 374 (1995) 113-116.

[8] C.C. Zouboulis, H. Seltmann, N. Hiroi, W. Chen, M. Young, M. Oeff, W.A. Scherbaum, C.E. Orfanos, S.M. McCann, S.R. Bornstein, Corticotropin-releasing hormone/ An autocrine hormone that promotes lipogenesis in human sebocytes, PNAS 99 (2002) 7148-7153.

[9] M. Bohm, M. Schiller, S. Stander, H. Seltmann, Z. Li, T. Brzoska, D. Metze, H.B. Schioth, A. Skottner, K. Seiffert, C.C. Zouboulis, T.A. Luger, Evidence for expression of melanocortin-1 receptor in human sebocytes in vitro and in situ, J. Invest. Dermatol. 118 (2002) $533-539$.
[10] M. Bohm, C.C. Zouboulis, T.A. Luger, Evidence for expression of mu-opoid receptors on human sebocytes which mediate paracrine and/or endocrine action of beta-endorphin (abstract), J. Invest. Dermatol. 119 (2002) 278.

[11] K. Seiffert, C.C. Zouboulis, H. Seltmann, R.D. Granstein, Expression of neuropeptide receptors by human sebocytes and stimulatory effect of their agonists on cytokine production (abstract), Horm. Res. 53 (2000) 102.

[12] C.C. Zouboulis, M. Bohm, Neuroendocrine regulation of sebocytes-a pathogenic link between stress and acne, Exp. Dermatol. 13 (Suppl. 4) (2004) 31-35.

[13] J.P. Leeming, K.T. Holland, W.J. Cunliffe, The microbial colonization of inflamed acne vulgaris lesions, Br. J. Dermatol. 118 (1988) 203-208.

[14] J.J. Leyden, K.J. McGinley, B. Vowels, Propionibacterium acnes colonization in acne and non-acne, Dermatology 196 (1988) 55-58.

[15] G.M. Graham, M.D. Farrar, J.E. Cruse-Sawyer, K.T. Holland, E. Ingham, Proinflammatory cytokine production by human keratinocytes stimulated with Propionibacterium acnes and P. acnes GroEL, Br. J. Dermatol. 150 (2004) 421-428.

[16] I. Nagy, A. Pivarcsi, A. Koreck, M. Szell, E. Urban, L. Kemeny, Distinct strains of Propionibacterium acnes induces selective human $\beta$-defensin-2 and interleukin-8 expression in human keratinocytes through Toll-like receptors, J. Invest. Dermatol. 124 (2005) 931-938.

[17] M. Schaller, M. Loewenstein, C. Borelli, K. Jacob, M. Vogeser, W.H. Burgdorf, G. Plewig, Induction of a chemoattractive proinflammatory cytokine response after stimulation of keratinocytes with Propionibacterium acnes and coproporphyrin III, Br. J. Derm. 153 (2005) 66-71.

[18] A. McDowell, S. Valanne, G. Ramage, M.M. Tunney, J.V. Glenn, G.C. McLorinan, A. Bhatia, J.F. Maisonneuve, M. Lodes, D.H. Persing, S. Patrick, Propionibacterium acnes types I and II represent phylogenetically distinct groups, J. Clin. Microbiol. 43 (2005) 326-334.

[19] S. Valanne, A. McDowell, G. Ramage, M.M. Tunney, G.G. Einarsson, S. O'Hagan, G.B. Wisdom, D. Fairley, A. Bhatia, J.F. Maisonneuve, M. Lodes, D.H. Persing, S. Patrick, CAMP factor homologues in Propionibacterium acnes: a new protein family differentially expressed by types I and II, Microbiology 151 (2005) 1369-1379.

[20] K. Takeda, S. Akira, Microbial recognition by Toll-like receptors, J. Dermatol. Sci. 34 (2004) 73-82.

[21] M.G. Netea, C. Van der Graaf, J.W. Van der Meer, B.J. Kullberg, Tolllike receptors and the host defense against microbial pathogens: bringing specificity to the innate-immune system, J. Leukoc. Biol. 75 (2004) $749-755$.

[22] C. Pasare, R. Medzhitov, Toll-like receptors: linking innate and adaptive immunity, Microbes Infect. 6 (2004) 1382-1387.

[23] M.K. Oeff, H. Seltmann, N. Hakiy, B. Bogdanoff, A. Nastos, R. Walters, S.R. Bornstein, C.C. Zouboulis, Toll-like Receptor 2 and 4-dependent regulation of inflammatory signaling in human sebocytes (abstract), J. Invest. Dermatol. 119 (2002) 736.

[24] P. Georgel, K. Crozat, X. Lauth, E. Makrantonaki, H. Seltmann, S. Sovath, K. Hoebe, X. Du, S. Rutshmann, Z. Jiang, T. Bigby, V. Nizet, C.C. Zouboulis, B. Beutler, A TLR2-responsive lipid effector pathway protects mammals against Gram-positive bacterial skin infections, Infect. Immun. 73 (2005) 4512-4521.

[25] S. Kumar, K. Tamura, M. Nei, MEGA3: Integrated software for molecular evolutionary genetics analysis and sequence alignment, Brief Bioinform 5 (2004) 150-163.

[26] J.D. Thompson, D.G. Higgins, T.J. Gibson, CLUSTAL W: improving the sensitivity of progressive multiple sequence alignment through sequence weighing, position-specific gap penalties and weight matrix choice, Nucleic Acids Res. 22 (1994) 4673-4680.

[27] C.C. Zouboulis, H. Seltmann, H. Neitzel, E. Orfanos, Establishment and characterization of an immortalized human sebaceous gland cell line (SZ95), J. Invest. Dermatol. 113 (1999) 1011-1020.

[28] A. Pivarcsi, L. Bodai, B. Rethi, A. Kenderessy-Szabo, A. Koreck, M. Szell, Z. Beer, Z. Bata-Csorgoo, M. Magocsi, E. Rajnavolgyi, A. Dobozy, L. Kemeny, Expression and function of Toll-like receptors 2 and 4 in human keratinocytes, Int. Immunol. 15 (2003) 721-730. 
[29] A. Pivarcsi, I. Nagy, A. Koreck, K. Kis, A. Kenderessy-Szabo, M. Szell, A. Dobozy, L. Kemeny, Microbial compounds induce the expression of pro-inflammatory cytokines, chemokines and human $\beta$-defensin- 2 in vaginal epithelial cells, Microbes Infect 7 (2005) 1117-1127.

[30] M.M. Tunney, S. Patrick, M.D. Curran, G. Ramage, D. Hanna, J.R. Nixon, S.P. Gorman, R.I. Davis, N. Anderson, Detection of prosthetic hip infection at revision arthroplasty by immunofluorescence microscopy and PCR amplification of the bacterial 16S rRNA gene, J. Clin. Microbiol. 37 (1999) 3281-3290.

[31] J.G.H. Dinulos, L. Mentele, L.P. Frederics, B.A. Dale, G.L. Darmstadt, Keratinocyte expression of human $\beta$ defensin 2 following bacterial infection: role in cutaneous host defense, Clin. Diagn. Lab. Immunol. 10 (2003) 161-166.

[32] H. Kurzen, H. Berger, C. Jager, W. Hartschuh, H. Naher, A. Gratchev, S. Goerdt, M. Diechmann, Phenotypical and molecular profiling of the extraneuronal cholinergic system of the skin, J. Invest. Dermatol. 123 (2004) 937-949.

[33] T. Mosmann, Rapid colorimetric assay for cellular growth and survival: application to proliferation and cytotoxicity assays, J. Immunol. Methods 65 (1983) 55-63.

[34] B. Dreno, F. Poli, Epidemiology of acne, Dermatology 206 (2003) 7-10.

[35] P.E. Mouser, B.S. Baker, E.D. Seaton, A.C. Chu, Propionibacterium acnes-reactive $\mathrm{T}$ helper- 1 cells in the skin of patients with acne vulgaris, J. Invest. Dermatol. 121 (2003) 1226-1228.

[36] K.J. McGinley, G.F. Webster, M.R. Ruggieri, J.J. Leyden, Regional variations in density of cutaneous propionibacteria: Correlation of Propionibacterium acnes populations with sebaceous secretion, J. Clin. Microbiol. 12 (1980) 672-675.

[37] U. Jappe, R. Boit, M.D. Farrar, E. Ingham, J. Sandoe, K.T. Holland, Evidence for diversity within Propionibacterium acnes: a comparison of the T-cell stimulatory activity of isolates from inflammatory acne, endocarditis and the laboratory, J. Eur. Acad. Dermatol. Venereol. 18 (2004) 450-454.

[38] J.L. Johnson, C.S. Cummins, Cell wall composition and deoxyribonucleic acid similarities among the anaerobic coryneforms, classical propionibacteria, and strains of Arachnia propionica, J. Bacteriol. 109 (1972) 1047-1066.

[39] J. Kim, M.T. Ochoa, S.R. Krutzik, O. Takeuchi, S. Uematsu, A.J. Legaspi, H.D. Brightbill, D. Holland, W.J. Cunliffe, S. Akira, P.A. Sieling, P.J. Godowski, R.L. Modlin, Activation of toll-like receptor 2 in acne triggers inflammatory cytokine responses, J. Immunol. 169 (2002) 1535-1541.

[40] A.H. Jeremy, D.B. Holland, S.G. Roberts, K.F. Thomson, W.J. Cunliffe, Inflammatory events are involved in acne lesion initiation, J. Invest. Dermatol. 121 (2003) 20-27.

[41] J. Harder, J. Bartels, E. Christophers, J.M. Schroder, A peptide antibiotic from human skin, Nature 387 (1997) 861.

[42] J.M. Schroder, Epithelial peptide antibiotics, Biochem. Pharmacol. 57 (1999) 121-134.

[43] O.E. Sorensen, D.R. Thapa, A. Rosenthal, L. Liu, A.A. Roberts, T. Ganz, Differential regulation of $\beta$-defensin expression in human skin by microbial stimuli, J. Immunol. 174 (2005) 4870-4879.

[44] D. Yang, O. Chertov, S.N. Bykovskaia, Q. Chen, M.J. Buffo, J. Shogan, M. Anderson, J.M. Schroder, J.M. Wang, O.M. Howard, J.J. Oppenheim, Beta-defensins: linking innate and adaptive immunity through dendritic and T cell CCR6, Science 286 (1999) 525-528.

[45] F. Niyonsaba, H. Ogawa, I. Nagaoka, Human beta-defensin-2 functions as a chemotactic agent for tumour necrosis factor-alpha-treated human neutrophils, Immunology 111 (2004) 273-281.
[46] C.M. Chronnell, L.R. Ghali, R.S. Ali, A.G. Quinn, D.B. Holland, J.J. Bull, W.J. Cunliffe, I.A. McKay, M.P. Philpott, S. Muller-Rover, Human beta defensin-1 and -2 expression in human pilosebaceous units: upregulation in acne vulgaris lesions, J. Invest. Dermatol. 117 (2001) $1120-1125$.

[47] M.P. Philpott, Defensins and acne, Mol. Immunol. 40 (2003) 457-462.

[48] A. Pivarcsi, I. Nagy, L. Kemeny, Innate immunity of the skin: how keratinocytes fight against pathogens, Curr. Immunol. Rev. 1 (2005) 29-42.

[49] Q. Chen, T. Koga, H. Uchi, H. Hara, H. Terao, Y. Moroi, K. Urabe, M. Furue, Propionibacterium acnes-induced IL-8 production may be mediated by NF- $\mathrm{B}$ a activation in human monocytes, J. Dermatol. Sci. 29 (2002) 97-103.

[50] R. Newton, I.M. Adcock, P.J. Barnes, Superinduction of NF-kappa B by actinomycin D and cycloheximide in epithelial cells, Biochem. Biophys. Res. Commun. 218 (1996) 518-523.

[51] R. Gillitzer, M. Goebeler, Chemokines in cutaneous wound healing, J. Leukoc. Biol. 69 (2001) 513-521.

[52] G. Michel, L. Kemeny, R.U. Peter, A. Beetz, C. Ried, P. Arenberger, T. Ruzicka, Interleukin-8 receptor-mediated chemotaxis of normal human epidermal cells, FEBS Lett. 305 (1992) 241-243.

[53] Y. Tomari, P.D. Zamore, Perspective: machines for RNAi, Genes Dev. 19 (2005) 517-529.

[54] R.N. Bhattacharjee, S. Akira, Toll-like receptor signaling: emerging opportunities in human diseases and medicine, Curr. Immunol. Rev. 1 (2005) 81-90.

[55] B. Vega-Diaz, A. Jonard, S. Michel, Regulation of human monocyte tolllike receptor 2 (TLR2) expression by adapalene (abstract), J. Eur. Acad. Dermatol. Venereol. 16 (2002) 123-124.

[56] P.T. Liu, S.R. Krutzik, J. Kim, R.A. Modlin, Cutting Edge: All-trans retinoic acid downregulates TLR2 expression and function, J. Immunol. 174 (2005) 2461-2470.

[57] M.D. Farrar, E. Ingham, K.T. Holland, Heat shock proteins and inflammatory acne vulgaris: molecular cloning, overexpression and purification of a Propionibacterium acnes GroEL and DnaK homologue, FEMS Microbiol. Lett. 191 (2000) 183-186.

[58] G.A. Whale, I.C. Sutcliffe, A.R. Morrisson, E.L. Pretswell, N. Emmison, Purification and characterization of lipoglycan macroamphiphiles from Propionibacterium acnes, Antonie Van Leeuwenhoek 86 (2004) 77-85.

[59] H. Bruggemann, A. Henne, F. Hoster, H. Liesegang, A. Wiezer, A. Strittmatter, S. Hujer, P. Durre, G. Gottschalk, The complete genome sequence of Propionibacterium acnes, a commensal of human skin, Science 305 (2004) 671-673.

[60] G.C. McLorinan, J.V. Glenn, M.G. McMullan, S. Patrick, Propionibacterium acnes wound contamination at the time of spinal surgery, Clin. Orthop. Relat. Res. 437 (2005) 67-73.

[61] C.C. Zouboulis, S. Nestoris, Y.D. Adler, M. Orth, C.E. Orfanos, M. Picardo, E. Camera, W.J. Cunliffe, A new concept for acne therapy: a pilot study with zileuton, an oral 5-lipoxygenase inhibitor, Arch. Dermatol. 139 (2003) 668-670.

[62] R. Skidmore, R. Kovach, C. Walker, J. Thomas, M. Bradshaw, J. Leyden, C. Powala, R. Ashley, Effects of subantimicrobial-dose doxycycline in the treatment of moderate acne, Arch. Dermatol. 139 (2003) 459-464.

[63] J.I. Ross, A.M. Snelling, E. Carnegie, P. Coates, W.J. Cunliffe, V. Bettoli, G. Tosti, A. Katsambas, J.I. Galvan Perez Del Pulgar, O. Rollmann, L. Torok, E.A. Eady, J.H. Cove, Antibiotic-resistant acne: lessons from Europe, Br. J. Dermatol. 148 (2003) 467-478.

[64] C.C. Zouboulis, J. Piquero-Martin, Update and future of systemic acne treatment, Dermatology 206 (2003) 37-53. 